\title{
Achados neurológicos, alterações sensoriais da função olfativa, gustativa e auditiva em pacientes com Covid-19: uma revisão literária
}

\author{
Neurological findings, sensory changes in the olfactory, taste and auditory function in \\ patients with Covid-19: a literary review
}

Hallazgos neurológicos, cambios sensoriales en la función olfactoria, sabor y auditorio en pacientes con Covid-19: una revisión literária

Diana Babini Lapa de Albuquerque Britto ${ }^{1 *}$, Mônyka Ferreira Borges Rocha1, Luis Filipi Souza de Britto Costa ${ }^{2}$, Carlos Fernando de Britto Costa Filho ${ }^{3}$, Bruno Mendes Tenorio ${ }^{4}$, Carina Scanoni Maia $^{1}$, Juliana Pinto de Medeiros ${ }^{1}$, Fernanda das Chagas Angelo Mendes Tenorio ${ }^{1}$.

\section{RESUMO}

Objetivo: descrever achados relatados na literatura de alterações sensoriais da função auditiva, olfativa e gustativa presentes em pacientes com Covid-19. Revisão Bibliográfica: Registros científicos têm apresentado alterações neurológicas em pacientes com Covid-19. Os distúrbios olfativos e gustativos têm sido prevalentes entre eles, afirmando que a súbita anosmia ou ageusia precisam ser reconhecidas pela comunidade científica como sintomas importantes para o rastreio da infecção. Quanto à função auditiva, sintomas primários como perda auditiva neurossensorial, além de alterações significativas de limiares de tons puros de alta frequência e amplitudes das emissões otoacústicas evocadas transientes foram identificados em pacientes com teste positivo para a Covid-19. Os estudos relatam que apesar de complicações audiológicas pelo novo coronavírus ser pouco mencionadas na literatura, estudos mais aprofundados sobre essas possíveis manifestações clínicas na Covid-19 é de extrema relevância afim auxiliar no diagnóstico precoce da doença. Considerações finais: Diante do exposto, podemos considerar que os sintomas auditivos, bem como de anosmia e ageusia podem se apresentar como a única manifestação da infecção pelo novo coronavírus sendo importante a investigação desses sintomas além do conhecimento a partir de novas pesquisas sobre os mecanismos patogênicos que levam ao envolvimento dessas afecções.

Palavras-chave: Neurologia, Anosmia, Ageusia, Audiologia, Covid-19.

\begin{abstract}
Objective: to describe findings reported in the literature of sensory changes in auditory, olfactory and gustatory function present in patients with Covid-19. Bibliographic Review: Scientific records have shown neurological changes in patients with Covid-19. Olfactory and gustatory disorders have been prevalent among them, stating that sudden anosmia or ageusia need to be recognized by the scientific community as important symptoms for screening for infection. As for auditory function, primary symptoms such as sensorineural hearing loss, in addition to significant changes in pure high-frequency tone thresholds and amplitudes of transient evoked otoacoustic emissions were identified in patients with a positive Covid-19 test. Studies report that although audiological complications due to the new coronavirus are rarely mentioned in the literature, further studies on these possible clinical manifestations in Covid-19 are extremely relevant in order to assist in the early diagnosis
\end{abstract}

\footnotetext{
${ }^{1}$ Universidade Federal de Pernambuco (UFPE), Recife - PE. *E-mail: diana.babini@gmail.com

2 USF Vicente Alberto Caricio Malvinas II, Jaboatão dos Guararapes - PE.

${ }^{3}$ Clínica Britto Fisio e Sport Club do Recife, Recife - PE.

${ }^{4}$ Universidade Federal da Paraíba (UFPB), João Pessoa - PB.
} 
of the disease. Conclusion: In view of the above, we can consider that the auditory symptoms, as well as anosmia and ageusia, may present themselves as the only manifestation of infection by the new coronavirus, and it is important to investigate these symptoms in addition to knowledge from new research on pathogenic mechanisms that lead to the involvement of these conditions.

Keywords: Neurology, Anosmia, Ageusia, Audiology, Covid-19.

\section{RESUMEN}

Objetivo: describir los hallazgos reportados en la literatura de cambios sensoriales en la función auditiva, olfativa y gustativa presentes en pacientes con Covid-19. Revisión bibliográfica: los registros científicos han mostrado cambios neurológicos en pacientes con Covid-19. Los trastornos olfativos y gustativos han prevalecido entre ellos, indicando que la comunidad científica debe reconocer la anosmia o ageusia repentina como síntomas importantes para detectar infecciones. En cuanto a la función auditiva, se identificaron síntomas primarios tales como pérdida auditiva neurosensorial, además de cambios significativos en los umbrales de tonos de alta frecuencia y amplitudes de emisiones otoacústicas evocadas transitorias en pacientes con una prueba Covid-19 positiva. Los estudios informan que, aunque las complicaciones audiológicas debido al nuevo coronavirus rara vez se mencionan en la literatura, otros estudios sobre estas posibles manifestaciones clínicas en Covid-19 son extremadamente relevantes para ayudar en el diagnóstico precoz de la enfermedad. Conclusión: en vista de lo anterior, podemos considerar que los síntomas auditivos, así como la anosmia y la ageusia pueden presentarse como la única manifestación de infección por el nuevo coronavirus y es importante investigar estos síntomas además del conocimiento de una nueva investigación sobre los mecanismos patogénicos que conducir a la participación de estas condiciones.

Palabras clave: Neurología, Anosmia, Ageusia, Audiologia, Covid-19.

\section{INTRODUÇÃO}

Desde o final de dezembro de 2019, um surto de pneumonia em Wuhan, na China, provocou uma atenção global (HUANG C, et al., 2020). Rapidamente a nova doença se alastrou pelo mundo em proporções aceleradas (PHELAN AL, et al., 2020). A doença pandêmica é atualmente conhecida como Covid-19, apresentando como agente patogênico o SARS-CoV-2.

Chen Y, et al. (2020) relatam que a Síndrome Respiratória Aguda Grave por Coronavírus 2 (SARS-CoV2) é um vírus de ácido ribonucleico (RNA) de fita única, o qual possui um genoma com sete genes. Os autores também afirmam que o SARS-CoV-2 apresenta em sua constituição proteínas estruturais, sendo as principais proteínas, as espículas (S1 e S2), a proteína de membrana (M), a proteína do envelope (E) e a proteína do nucleocapsídeo $(\mathrm{N})$.

A presença da SARS-CoV e seu vírus RNA foi detectado em células dos pulmões e outros órgãos do corpo humano. Órgãos esses que possuem a presença da enzima conversora da angiotensina 2 (ECA2) (HE L, et al., 2006). Esse achado indica que essas células são os principais ativos da infecção por SARS-CoV em humanos sendo a ECA2 o seu receptor funcional (LI WH, et al., 2003).

Shen C, et al. (2020) encontraram uma diversidade de variantes virais em alguns pacientes infectados, que é sugestivo da rápida evolução da SARS-CoV-2. Isso acontece pelo fato de, por ser um vírus de RNA, o risco de recombinação ativa e mutações, que são atribuíveis às polimerases de RNA dependentes de RNA, acontece de forma mais acelerada. Huang C, et al. (2020) afirmam que o coronavírus (CoVs), de uma forma geral, podem infectar tanto animais como humanos, e as infecções podem afetar o sistema respiratório, gastrointestinal e o sistema nervoso central (SNC). Esses fatores contribuem para que o vírus consiga se tornar cada vez mais nocivo.

É importante salientar que o espectro da infecção do SARS-CoV-2 se apresenta de maneira ampla, englobando desde a infecção assintomática, doença leve do trato respiratório superior e pneumonia viral grave com insuficiência respiratória, podendo evoluir para óbito (GORBALENYA AE, et al., 2020). 
O coronavírus é de fácil transmissão e acomete de forma mais grave pacientes portadores de debilidades crônicas. Doentes pulmonares, doentes renais crônicos, diabéticos, hipertensos, cardiopatas, usuários de corticoides ou drogas imunossupressoras, tabagistas e idosos, correspondem à parte da população mais susceptível à pneumonia (WU Z e MCGOOGAN JM, 2020). Os sintomas clássicos da infecção do SARSCoV-2 são: febre, tosse seca e falta de ar, afirmam Kowalski LP, et al. (2020), aparecendo normalmente entre ○ $2^{\circ}$ e $014^{\circ}$ dia de infecção.

O Ministério da Saúde (2020) relata que indivíduos infectados pelo Covid-19 também podem apresentar outros sintomas além dos referidos acima como: produção de escarro, congestão nasal ou conjuntival, dificuldade para deglutir, dor de garganta, coriza, saturação de $\mathrm{O} 2<95 \%$, sinais de cianose, batimento de asa de nariz, tiragem intercostal e dispneia. Outros sinais e sintomas inespecíficos como: fadiga, mialgia/artralgia, cefaleia, calafrios, linfonodomegalia, diarreia, náusea, vômito, desidratação e inapetência também podem estar presentes. Nas últimas semanas têm surgido diversos relatos de casos com quadro neurológicos como encefalite mielite, crises convulsivas e até estados epilépticos refratárias. O estudo alerta para atenção a novas manifestações associadas a Covid-19 (PONTES NETO OM, 2020).

Kowalski LP, et al. (2020) afirmam que relatórios recentes em todo o mundo mostraram que anosmia e ageusia são sintomas significativos associados à pandemia de Covid-19. Além disso, casos de alterações da função auditiva já foram relatados após infecção pelo vírus (MUSTAFA MWM, 2020). Diante do exposto, o presente estudo tem como objetivo descrever achados relatados na literatura científica sobre achados neurológicos, alterações sensoriais da função auditiva, olfativa e gustativa presentes em pacientes infectados por Covid-19.

\section{REVISÃO BIBLIOGRÁFICA}

\section{Achados neurológicos na Covid-19}

Cheng $Q$, et al. (2020) relatam que, embora os principais sintomas do Covid-19 estejam sendo gerados pelo acometimento do sistema respiratório, em alguns casos de Covid-19 confirmados estão aparecendo também sintomas neurológicos, o que vem despertando a preocupação sobre o possível potencial de invasão intracraniana e manifestações neurológicas que esse vírus tem, seja na fase aguda ou longo prazo. Além disso, o estudo citado acima afirma que não está claro na literatura até que ponto o SARS-CoV-2 está presente no cérebro e, nesse caso, seu papel patogênico no SNC. Sabe-se que há evidências de neuroinvasão e neurovirulência de hCoVs em estudos com animais e humanos (CHENG Q, et al., 2020)

Cheng Q, et al. (2020) ainda relatam que os hCoVs incluem sete cepas, três das quais, nomeadamente SARS-CoV-2, SARS-CoV e coronavírus relacionado à síndrome respiratória do Oriente Médio (MERS-CoV), podem causar doenças respiratórias graves com uma morbidade e mortalidade relativamente altas. Como 0 SARS-CoV-2 pertence à mesma família e compartilha características em termos de propriedades de ligação ao receptor, tem-se destacado, cada vez mais, a necessidade de exploração sobre sua possível manifestação no SNC.

Estudos recentes vêm sendo realizados intensivamente no atual cenário pandêmico, apresentando relatos de casos com quadro neurológicos como encefalite mielite, crises convulsivas estados epilépticos, alertando a população científica quanto às novas manifestações associadas à infecção pelo Covid-19 (PONTES NETO $\mathrm{OM}, 2020)$.

Em relação ao sistema nervoso, pesquisadores da Universidade Estadual de Campinas (UNICAMP), confirmaram recentemente que o SARS-CoV-2 é capaz de infectar neurônios humanos. Conforme Martinsde-Souza D (2020), a comprovação ocorreu através da realização de experimentos in vitro em cultura de células. Mesmo sendo recente para conclusões clínicas, o pesquisador afirma que a infecção dos neurônios traz maiores riscos ao paciente, como é o caso do aparecimento de sintomas neurológicos como a anosmia - perda de olfato.

De acordo com Cheng Q, et al. (2020), o SARS-CoV-2 também pode usar ECA2 como receptor celular. No entanto, a falta da expressão dessa enzima nas células neuronais, tanto a nível da transcrição quanto a 
nível da proteína, contrasta com a suscetibilidade viral dessas células bem como a citopatogenicidade durante a fase aguda da infecção por tanto nas células neurais (YAMASHITA M, et al., 2005) como no cérebro humano (HAMMING I, et al., 2004).

Recentemente, um estudo publicado Mao L, et al. (2020) relatou manifestações neurológicas no atual surto pandêmico do Covid-19, o qual envolveu 214 pacientes confirmados com Covid-19. Deste quantitativo, 36,4\% (78) dos pacientes apresentaram manifestações neurológicas, o que confirma a lógica do potencial neurotrópico no vírus COVID-19 (BAIG AM, et al., 2020).

Pelliteroa SE e Ferrer-Berguab G (2020) relataram o caso de uma mulher de 30 anos, com teste positivo para o novo coronavírus, que apresentou sintomas neurológicos como única manifestação da doença. $A$ paciente referiu sensação de instabilidade e perda de equilíbrio de 48 horas de progressão, com piora desses sintomas ao levantar-se, necessitando de auxílio para deambular. O exame físico confirmou a presença de disfunção vestibular aguda provavelmente devido à COVID-19, após ser descartado outras etiologias possíveis.

O estudo supracitado ainda infere que o curso clínico observado na paciente pode sugerir que o vírus tenha acessado diretamente o sistema nervoso atravessando o epitélio olfativo pela placa cribiforme etmoidal, explicando a presença da anosmia e dos sintomas vestibulares. Essa disseminação na circulação sistêmica durante a fase inicial ou até mesmo posterior a infecção, pode levar ao envolvimento cerebral, uma vez que a presença do Covid-19 na circulação permite com que o vírus passe para a circulação cerebral. Baig $A$, et al. (2020) afirmam que uma vez o vírus localizado em meio aos tecidos neuronais, sua interação com os receptores ECA2, expressa em neurônios pode promover um ciclo de brotamento viral acompanhado de danos neuronais, como foi visto em casos de SARS-CoV no passado.

Pelliteroa SE e Ferrer-Berguab G (2020) ainda enfatizam a importância de estarmos cientes de que os sintomas neurológicos podem se apresentar como a única manifestação da infecção por SARS-CoV-2, sendo essencial a garantia do diagnóstico precoce da infecção em pacientes com sintomas neurológicos agudos no contexto de uma epidemia, a fim de tomar ações para impedir a transmissão da doença.

\section{Alterações sensoriais das funções olfativa e gustativa na Covid-19}

Pellegrini G, et al. (2005) afirmam que tanto o olfato como o paladar são sentidos químicos. Os sistemas neurais que intermedeiam estas sensações - sistemas gustativo e olfatório - estão entre aqueles filogeneticamente mais antigos do encéfalo e ao perceberem substâncias químicas na cavidade oral e nasal trabalham conjuntamente.

As sensações surgem pela interação de moléculas com os receptores da olfação e gustação e tais impulsos se propagam para o sistema límbico, bem como para as áreas corticais superiores (TORTORA GJ e GRABOWSKI SR, 2005).

Riel DV, et al. (2015) descrevem que o nervo olfatório possui neurônios receptores olfativos que conectam diretamente a cavidade nasal ao sistema nervoso central (SNC), uma vez que esses neurônios olfativos estendem seus axônios através da placa cribriforme do osso etmóide até o bulbo olfativo do cérebro, o que pode, desta forma, usar o nervo olfatório como atalho ao SNC. Ollarves-Carrero MA, et al. (2020) afirmam que tanto os distúrbios olfativos como os distúrbios de paladar estão relacionados a uma ampla gama de infecções virais. Desta forma, os múltiplos vírus tanto podem usar os nervos neurológicos como também acometer o SNC, causando distúrbios olfativos (RIEL DV, et al., 2015).

Anosmia, em particular, tem sido observado em pacientes com resultado positivo para o coronavírus sem outros sintomas. Por esse motivo, a Academia Americana de Otorrinolaringologia - AAO-HNSF (2020) propôs que esses sintomas fossem adicionados à lista de ferramentas de rastreamento para possível infecção por Covid-19. Em 17 de abril de 2020, os Centros de Controle e Prevenção de Doenças (2020) acrescentaram

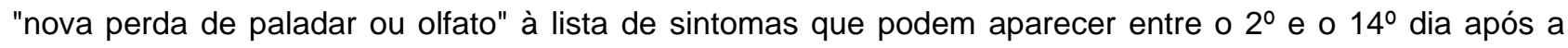
exposição ao vírus. 
Gilania S, et al. (2020) relatam que com o aparecimento do Covid-19 em Teerã, no Irã, houve um aumento de registros de perda olfativa completa - anosmia. Lechien JR, et al. (2020) afirmam que os distúrbios olfativos e gustativos, são prevalentes entre os pacientes europeus com Covid-19.

Vaira LA, et al. (2020a) descreveu em seu estudo que a função olfativa e gustativa foi testada objetivamente em 72 pacientes tratados com Covid-19 num Hospital Universitário de Sassari. Como resultados, $73,6 \%$ dos pacientes relataram ter ou ter tido distúrbios quimiossensíveis. A avaliação olfativa mostrou redução da sensibilidade (hiposmia) de grau variável em 60 casos e anosmia em 2 pacientes. A avaliação gustativa revelou redução da sensibilidade (hipogeusia) em 33 casos e ageusia em 1 paciente. Desta forma, o estudo afirma que tanto as disfunções olfativas quanto as gustativas representam achados clínicos comuns no Covid-19, Vale salientar que não havia alterações de rinite correlacionadas aos pacientes infectados.

Yan $\mathrm{CH}$, et al. (2020) realizaram um estudo com um total de 1.480 pacientes, todos com sintomas semelhantes. Os pacientes foram submetidos ao teste Covid-19 e capturou 59 de 102 (58\%) pacientes positivos e 203 de 1378 (15\%) negativo para a infecção. Traçando um comparativo entre as populações, a perda de olfato e paladar foi relatada respectivamente com $68 \%$ e $71 \%$ dos indivíduos com teste positivo. Já nos pacientes com teste de Covid-19 negativo, a presença de perda de olfato e paladar foi relatada em $16 \%$ e $17 \%$ dos indivíduos, respectivamente. O comprometimento do olfato e do paladar foi associado de forma independente e forte à positividade do Covid-19.

Este estudo demonstra a prevalência e apresentação única de comprometimento quimiossensorial em Covid-19-positivos em comparação com Covid-19-negativos. Encontramos uma associação significativa entre perda de paladar e olfato com a infecção por Covid-19-positivos (YAN CH, et al., 2020)

$\mathrm{Xu} \mathrm{H}$, et al. (2020) afirmam que a cavidade oral pode atuar como porta de entrada para infecções e consequentemente levar ao desenvolvimento da ageusia. Além disso, Aziz M, et al. (2020) descrevem que o mecanismo da SARS-CoV-2 que pode ocasionar alteração do paladar é a sua capacidade em se conectar à enzima ECA2, que é rapidamente expressa em vários sistemas orgânicos, incluindo a mucosa da cavidade oral e principalmente na superfície da língua.

O papel dessa enzima na modulação da percepção do paladar foi destacado em muitos estudos que analisam os efeitos colaterais quimiossensíveis de inibição da ECA2 e bloqueadores da angiotensina II (TSURUOKA S, et al., 2005).

Outra informação relevante trazida por Vaira LA, et al. (2020b), é que o ácido siálico é um componente fundamental da mucina salivar. Ele protege as glicoproteínas que transportam moléculas gustativas no interior dos poros do paladar devido à degradação enzimática prematura.

Desta forma, Vaira LA, et al. (2020b) afirmam que o SARS-CoV-2 pode, portanto, ocupar os locais de ligação do ácido siálico no paladar, acelerando, assim, a degradação das partículas gustativas. Por fim, uma outra hipótese é que a capacidade de percepção dos sabores em indivíduos com Covid-19 seja afetada em decorrência da presença concomitante de distúrbios olfativos, em consequência da intrínseca relação entre esses dois sistemas quimiosensoriais.

O potencial neuro invasivo da SARS-CoV-2 pode ter um papel na fisiopatologia da anosmia e ageusia. Como a mucosa olfativa está localizada na região superior da cavidade nasal, um efeito direto ou indireto da SARS-CoV-2 pode ser outra explicação para esses sintomas (BÉNÉZIT F, et al., 2020).

\section{Alteração sensorial da função auditiva na Covid-19}

Tendo em vista que a Covid-19 é uma doença infecciosa causada por um novo coronavírus recémdescoberto, estudos têm investigado com veemência as características clínicas desenvolvidas pelos pacientes infectados pelo agente patógeno SARS-CoV-2, sendo observado vários cenários sintomatológicos que tais indivíduos podem seguir no curso da doença. Um destes cenários é caracterizado por alterações da função auditiva. Sabe-se que várias infecções virais podem causar danos do sistema auditivo e 
consequentemente gerar alterações na função de auditiva que podem ser permanentes ou temporárias (MARTINS OR, 2017).

Considerando a perda auditiva uma das alterações mais comuns desenvolvidas em casos de infecção viral, estas podem ser unilateral ou bilateral, e de grau leve a profundo, sendo tipicamente sensorial, apesar de perdas auditivas condutivas e mistas serem observadas em alguns casos (VIEIRA ABC, et al., 2010). Embora várias infecções virais possam provocar alterações da função auditiva, tais manifestações apresentadas em indivíduos com a Covid-19 começaram a ser investigadas quanto a sua característica, motivo, causa e prevalência (MUSTAFA MWM, 2020; SRIWIJITALAI W e WIWANITKIT V, 2020).

A corrida rápida por estratégias antivirais específicas no combate ao novo coronavírus, como várias opções terapêuticas para o tratamento e prevenção desta doença tem sido evidenciada nos cenários das pesquisas realizadas em meio à pandemia. Desta forma, a administração de medicamentos antivirais em pacientes infectados pelo SARS-CoV-2 está sendo realizada, sendo alguns desses medicamentos, Favipiravir, Remdesivir, Lopinavir, Ritonavir, Interferon peguilhado com Ribavirina, Cloroquina e Hidroxicloroquina (AHN DG, et al., 2020).

É importante expor que algumas dessas substâncias, apesar de ser citado como antivirais promissores no tratamento dos pacientes infectados, o uso de alguns fármacos, como por exemplo, os antimaláricos, cloroquina e hidroxicloroquina, já foram associados a alterações auditivas em humanos. Tais alterações são relatadas devido à afinidade desses componentes pelas células portadoras de melanina, encontradas no ouvido interno. (CHATELET JN, et al., 2016; FIGUEIREDO MC, et al., 2004). Diante disso, presumisse que possam existir inter-relações entre perda auditiva e Covid-19, além de possíveis associações de manifestações auditivas com o uso de medicamentos antivirais no tratamento dessa infecção.

Em um estudo de relato de caso, uma mulher idosa apresentou como sintoma primário a perda auditiva neurossensorial sem observação de mudança do quadro. Os autores relataram que apesar das complicações audiológicas por coronavírus ser pouco mencionadas na literatura, estudos mais aprofundados sobre essas possíveis manifestações clínicas na Covid-19 é de extrema relevância (SRIWIJITALAI W e WIWANITKIT V, 2020).

Mustafa MWM (2020) relatou o perfil audiológico de vinte casos assintomáticos e positivos para a Covid19 e comparou os resultados dos exames auditivos com um grupo de indivíduos de audição normal e com ausência da infecção viral. Os resultados demonstraram limiares de tons puros de alta frequência e amplitudes das emissões otoacústicas evocadas transientes significativamente piores no grupo de indivíduos infectados pelo novo coronavírus.

No estudo supracitado, as alterações observadas no grupo de infectados foi atribuída à possíveis efeitos deletérios da infecção viral nas células ciliadas externas cocleares, mencionando que o mecanismo desses efeitos necessita de mais pesquisas. Foi referido ainda que a ausência de sintomas principais pode ocultar um impacto desconhecido nos sutis órgãos sensoriais, tomando a cóclea como exemplo (MUSTAFA MWM, 2020).

Em uma nota de opinião sobre a perda auditiva e a Covid-19, Cure E, Cure MC (2020) descreveu como essa complicação muito rara em pacientes infectados pode se desenvolver. Desta forma, os autores mencionam que após a entrada do vírus pelas vias aéreas e sua penetração nos pulmões por meio da ECA2, ele se liga à cadeia beta da hemoglobina e penetra no eritrócito, podendo ser transportado por estes e possivelmente infectando todos os tecidos que a possuem. Considerando a alta quantidade de enzima ECA2 no cérebro e no bulbo, o centro auditivo localizado no lobo temporal pode sofrer danos com o aumento da carga viral.

Além disso, o estudo citado acima infere que, quando o vírus infecta os eritrócitos, ocasionando sua desoxigenação, se houver uma ativação excessiva do vírus no centro auditivo do cérebro, pode existir a possibilidade ter causado hipóxia e consequentemente lesão do centro auditivo (CURE E, CURE MC, 2020).

Estudos sobre a relação de alteração da função auditiva com o uso de medicamentos no tratamento de pessoas com Covid-19 ainda não foram descritas na literatura, porém, é importante conhecer sobre essas possíveis afecções associadas ao uso de antivirais nesses indivíduos. 
Devemos também estar cientes de que os sintomas auditivos podem se apresentar como a única manifestação da infecção pelo novo coronavírus. No entanto, os mecanismos patogênicos que levam o envolvimento dessas afecções em pacientes com infecção pelo SARS-CoV-2 não são bem definidos, sendo importante a realização de novas pesquisas com essa temática, a fim de garantir o diagnóstico precoce da doença.

\section{CONSIDERAÇÕES FINAIS}

Os dados expostos nessa revisão permitem o conhecimento de evidências a respeito de novas manifestações clínicas da infecção em humanos pela Covid-19, sendo os sintomas de anosmia e ageusia comumente presentes nos indivíduos testados positivos para a doença, enquanto que os sintomas de alterações neurológicas e da função auditivas podem apresentar-se em um espectro menor e como único sintoma da infecção. Desta forma, esta revisão endossa a importância de novas pesquisas na investigação das diferentes manifestações sintomatológicas na infecção pelo vírus SARS-CoV-2 com a finalidade de definir o amplo espectro de sintomas da doença e assegurando o diagnóstico precoce dos infectados pelo novo coronavírus.

\section{REFERÊNCIAS}

1. AHN DG, et al. Curret status of epidemiology, diagnosis, therapeutics, and vaccines for novel coronavirus disease 2019 (COVID-19). J Microbiol Biotechnol, 2020; 30(3): 313-324.

2. AZIZ M, et al. Taste Changes (Dysgeusia) in COVID-19: A systematic review and metaanalysis. Gastroenterology, 2020.

3. BAIG A, et al. Evidence of the COVID-19 Virus Targeting the CNS: Tissue Distribution, Host-Virus Interaction, and Proposed Neurotropic Mechanisms. ACS Chem Neurosci, 2020; 11: 995-998.

4. BÉNÉZIT F, et al. Utility of hyposmia and hypogeusia for the diagnosis of COVID-19. The Lancet Infectious Diseases, 2020.

5. BRASIL, MINISTÉRIO DA SAÚDE. Boletim epidemiológico Doença pelo novo coronavírus (COVID-19). Disponível em: https://www.saude.gov.br/noticias/agencia-saude. Acesso em: 16 abr. 2020.

6. CHATELET JN, et al. Hydroxychloroquine-induced hearing loss: First case of positive rechallenge and analysis of the French pharmacovigilance database. La Revue de Medecine Interne, 2016; 38(5): 340-343.

7. CHEN Y, et al. Emerging coronaviruses: Genome structure, replication, and pathogenesis. J Med Virol., 2020; 92(4): 418-423.

8. CHENG Q, et al. Infectivity of human coronavírus in the brain. EBioMedicine, 2020.

9. CURE E, CURE MC. Comment on "Hearing loss and COVID-19: A note". Am J Otolaryngol, 2020.

10. FIGUEIREDO MC, et al. Antimaláricos e Ototoxicidade. Rev Bras Reumatol, 2004; 44(3): 212-214

11. GILANIA S, et al. COVID-19 and anosmia in Tehran, Iran. Medical Hypotheses, 2020; 141: 109757.

12. GORBALENYA AE, et al. Severe acute respiratory syndrome-related coronavirus: the species and its viruses-a statement of the Coronavirus Study Group. Nature Microbiology, 2020.

13. HAMMING I, et al. Distribuição tecidual da proteína ACE2, o receptor funcional do coronavírus SARS. Um primeiro passo para entender a patogênese da SARS. J Pathol, 2004; 203: 631-637.

14. HE L, et al. Expression of elevated levels of pro-inflammatory cytokines in SARS-CoV-infected ACE2+ cells in SARS patients: relation to the acute lung injury and pathogenesis of SARS. J Pathol., 2006; 210(3): 288-297.

15. HUANG C, et al. Clinical features of patients infected with 2019 novel coronavirus in Wuhan, China. The Lancet, 2020; 395(10223): 497-506.

16. KOWALSKI LP, et al. COVID-19 pandemic: effects and evidence-based recommendations for otolaryngology and head and neck surgery practice. Head Neck Actions, 2020.

17. LECHIEN JR, et al. Olfactory and gustatory dysfunctions as a clinical presentation of mild-to-moderate forms of the coronavirus disease (COVID-19): a multicenter European study. European Archives of Oto-Rhino-Laryngology, 2020.

18. LI WH, et al. Angiotensin-converting enzyme 2 is a functional receptor for the SARS coronavirus. Nature, 2003; 426(6965): 450-454.

19. MAO L, et al. Neurological Manifestations of Hospitalized Patients with COVID-19 in Wuhan, China: a retrospective case series study. MedRxiv, 2020.

20. MARTINS OR, et al. Achados otológicos em pacientes pós-infecção pelo Zika vírus: estudos de caso. AudiologyCommunication Research, 2017; 22.

21. MARTINS-DE-SOUZA D. Estudo mostra que novo coronavírus consegue infectar neurônios humanos. Folha de Vitória. Disponível em: https://www.folhavitoria.com.br/saude/noticia/04/2020/estudo-mostra-que-novo-coronavirusconsegue-infectar-neuronios-humanos. Acesso em: 27 mai. 2020.

22. MUSTAFA MWM. Audiological profile of asymptomatic Covid-19 PCR-positive cases. Am J Otolaryngol, 2020. 
23. OLLARVES-CARRERO MA, et al. Anosmia in a healthcare worker with COVID-19 in Madrid, Spain. Travel Medicine and Infectious Disease, 2020.

24. PELLEGRINI G, et al. A percepção do gosto salgado em indivíduos com e sem obstrução nasal. Rev. CEFAC, 2005; 7(3): 311-317.

25. PELLITEROA SE, FERRER-BERGUAB G. Report of a patient with neurological symptoms as the sole manifestation of SARS-CoV-2 infection Neurología, 2020.

26. PHELAN AL, et al. The novel coronavirus originating in Wuhan. China: Challenges for Global Health Governance. JAMA, 2020; 323(8): 709-710.

27. PONTES NETO, OM. Crescem relatos de manifestações neurológicas da covid-19. Jornal da USP. Disponível em: https://jornal.usp.br/radio-usp/crescem-relatos-de-manifestacoes-neurologicas-da-covid-19/. Acesso em: 28 mai. 2020.

28. RIEL DV, et al. The olfactory nerve: a shortcut for influenza and other viral diseases into the central nervous system. J Pathol, 2015; 235: 277-287.

29. SHEN C, et al. Treatment of 5 Critically III Patients With COVID-19 With Convalescent Plasma. JAMA, 2020; 323(16): 1582-1589.

30. SRIWIJITALAI W, WIWANITKIT V. Hearing loss and COVID-19: a note. Am J Otolaryngol, 2020.

31. TORTORA GJ, GRABOWSKI SR. Corpo Humano: Fundamentos de Anatomia e Fisiologia. $6^{\mathrm{a}}$ ed. Porto Alegre (RS): Artmed, 2005; 718p.

32. TSURUOKA S, et al. Comparative study of taste dirsurbance by losartan and perindopril in healthy volunteers. J Clin Pharmacol, 2005; 45(11): 1319-1323.

33. VAIRA LA, et al. Objective evaluation of anosmia and ageusia in COVID-19 patients: a single-center experience on 72 cases. Head \& Neck, 2020.

34. VAIRA LA, et al. Potential pathogenesis of ageusia and anosmia in COVID-19 patients. What we know from the literature. Int Forum of Allergy \& Rhinology, 2020.

35. VIEIRA ABC, et al. Doenças infecciosas e perda auditiva. Rev Med Minas Gerais, 2010; 20(1): 102-106.

36. WU Z, MCGOOGAN JM. Characteristics of and Important Lessons from the Coronavirus Disease 2019 (COVID-19) Outbreak in China: Summary of a Report of 72314 Cases from the Chinese Center for Disease Control and Prevention. JAMA, 2020.

37. XU H, et al. High expression of ACE2 receptor of 2019-nCoV on the epithelial cells of oral mucosa. Int $\mathrm{J}$ Oral Sci, 2020; 12: 1-5.

38. YAMASHITA M, et al. Suscetibilidade de linhas celulares neurais humanas e de ratos à infecção por SARScoronavírus. Biochem Biophys Res Commun, 2005; 334: 79-85.

39. YAN CH, et al. Association of Chemosensory Dysfunction and Covid-19 in Patients Presenting with Influenza-like Symptoms. International Forum of Allergy \& Rhinology, 2020. 